\title{
Von Hygiene- und Motivationsfaktoren: Einsichten von „Down Under"
}

\section{Kommentar zu „Qualifikationsprofil für Universitätsprofessoren der Wirtschaftsinformatik"}

DOI 10.1007/s11576-013-0372-4

\section{Der Autor \\ Prof. Dr. Jan Recker ( $\varangle)$ \\ Woolworths Chair of Retail \\ Innovation \\ Information Systems School \\ Queensland University of Technology \\ Brisbane, QLD \\ Australien \\ j.recker@qut.edu.au \\ Online publiziert: 2013-07-02 \\ This article is also available in English via http://www.springerlink.com and http://www.bise-journal.org: Recker J (2013) Of Hygiene and Motivator Factors: Views from "Down Under". Comments on "Qualification Profile of University Professors in Business and Information Systems Engineering (BISE)". Bus Inf Syst Eng. doi: 10.1007/ s12599-013-0277-1. \\ (C) Springer Fachmedien Wiesbaden 2013}

Ich habe jetzt fast zehn Jahre an einer australischen Universität verbracht, aber meine ersten Sporen in der Wirtschaftsinformatikforschung habe ich mir als wissenschaftlicher Mitarbeiter an der Universität Münster verdient. Obwohl ich mich (geografisch gesehen) fast am weitesten entfernt von dem Deutschen Universitätssystem aufhalte, habe ich doch Beziehungen und ein Interesse an der Entwicklung der Wirtschaftsinformatik-Disziplin in meinem Heimatland aufrecht erhalten.

Natürlich sind die Dinge in Australien anders - allerdings nicht notwendigerweise „auf dem Kopf“. Auch in Australien sind Akademiker unter starkem $\mathrm{Pu}$ blikationszwang, um sich eine der immer wenig werdenden Fakultätsstellen $\mathrm{zu}$ sichern. Es gibt Auseinandersetzungen über Journalrankings und Publikationen (Lamp 2009), und die Diskussi- on über Rigorosität versus Relevanz wird auch hier innig debattiert (Recker et al. 2009).

Dennoch gibt es einige Unterschiede, die für die Debatte über Professorenqualifikationen (Loos et al. 2013) relevant sind. Die auffälligsten Unterschiede im professoralen Karriereweg in Australien sind einerseits das Tenure-Track-System und andererseits die Organisation der Lehrbereiche und Forschungsabteilungen, die nicht nach Lehrstühlen erfolgt (obwohl es auch diese gibt - mit einer anderen Bedeutung), sondern in Gruppen von Akademikern mit verschiedenen Rängen. In diesem System könnten theoretisch alle Forscher Professoren sein (wenn sie die Anforderungen erfüllen) oder aber keiner wird Professor und stattdessen verweilen alle auf dem Level Lecturer, Senior Lecturer oder Associate Professor. Dies hängt von der individuellen Leistung, aber auch von Motivation und Lebenszielen ab. An unserem Institut gibt es mehrere Professoren, von denen einer die Rolle als Institutsleiter innehat, welche üblicherweise im RoundRobin-Schema wechselt. Andere Institute in Australien werden von Associate Professors geleitet. Ich kenne mehrere Kollegen, die keine Professur anstreben, vor allem, weil der höhere Rang weitere Anforderungen mit sich bringt wie zum Beispiel Leitung und Strategieentwicklung, zusätzlich zu Lehr-, Forschungs- und Serviceaufgaben, was nicht jedermanns Sache ist.

Ein anderer Kernunterschied liegt in der Trennung zwischen der Ernennung zum (Full) Professor und der Anerkennung der "Tenure“, also der Verdauerung der Stelle (analog der Verbeamtung auf Lebenszeit). Oft wird vermutet, dass Ernennung und Verdauerung einhergehen; zum Beispiel wenn ein junger Senior Lecturer nach vielen harten postdoktoralen Jahren gleichzeitig zum Associate Professor ernannt wird und eine Dauerstelle bekommt. Nach meiner Erfahrung sieht dies in der Realität anders aus. Tenure ist üblicherweise die Hauptsorge von Akademikern. Die Entscheidungen über Ernennung und Tenure sind nicht nur entkoppelt, sondern komplett eigenständige Prozesse mit unterschiedlichen Kriterien. Die Ernennung zum Professor ist das forschungstechnische „Ende“ einer Karriereleiter, die über verschiedene Ränge erfolgt, welche verschiedene Kriterienstaffelungen mit sich bringen.

Im Hinblick auf die Professorenqualifikation habe ich die Diskussion von Loos et al. (2013) über existierende und erwünschte Qualifikationskriterien als sehr hilfreich empfunden, um Struktur in ein zugegebenermaßen schlecht strukturiertes Entscheidungsproblem zu bringen. Aus meiner Sicht sind die meisten der debattierten Kriterien (z. B. eine Publikationsliste, Drittmittelakquisition, Lehrportfolio und Industrieverbindungen) stets relevant. Auf welche sollten sich junge Akademiker also fokussieren?

Ich habe es immer als hilfreich empfunden, zwischen Hygiene- und Motivationsfaktoren (Herzberg 1966) zu unterscheiden, bspw. um zu verstehen, wie Artikel geschrieben werden müssen, die zur Präsentation oder Publikation angenommen werden (Rosemann et al. 2010). Ich finde, sie sind nicht nur nützlich zur Differenzierung zwischen „Muss“ und „Kann“, sondern auch zum Verständnis, wie viel Aufwand auf jedes Kriterium verwenden werden sollte, nachdem ein gewisses Level erreicht wurde. Auf Basis dieser Unterteilung ist meine Sicht auf einige der relevanten Hygiene- und Motivationsfaktoren wie folgt (Abb. 1).

- Starke Publikationsleistung inklusive einer Auswahl von signifikanten Zeitschriftenbeiträgen ist eine notwendige, aber nicht hinreichende Anforderung geworden. Im Hinblick auf die hohe Zahl junger Akademiker im Vergleich zu verfügbaren Positionen und im Hinblick auf die zunehmende Relevanz von Forschungsexzellenz ist es wahrscheinlich, dass irgendeine Art der Publikations- (Dean et al. 
Abb. 1 Hygiene- und Motivationsfaktoren für eine Professor in Wirtschaftsinformatik

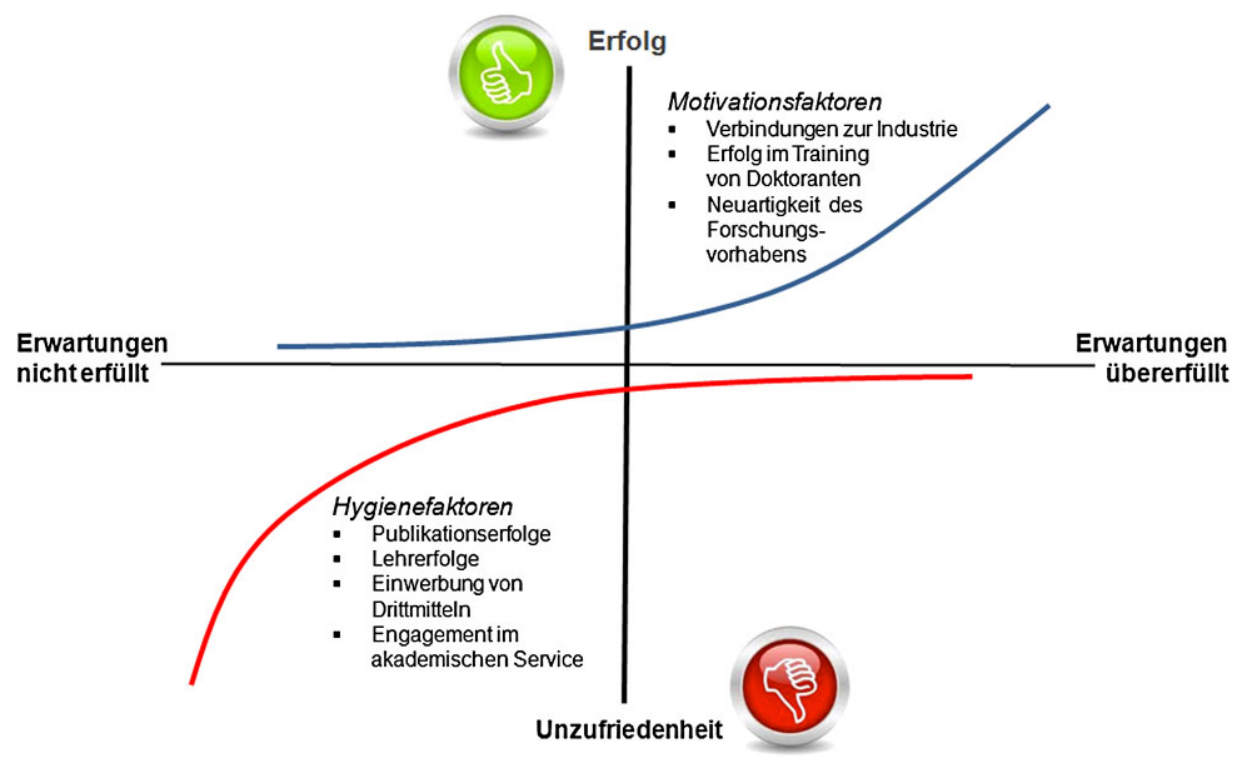

2011) oder Impact-Kennzahlen (Harzing 2013) auch weiterhin zum Vergleich von Individuen oder Institutionen verwendet wird. Aber wird es wichtig sein, wie viele Publikationen ein Antragssteller über eine gewisse erforderliche Anzahl (10 Zeitschriftenbeiträge? 50 Konferenzbeiträge?) hat? Wahrscheinlich nicht. Ich neige $\mathrm{zu}$ der Aussage, dass der „nächste Zeitschriftenbeitrag nicht so relevant sein wird wie der letzte“; das heißt, der Grenznutzen nimmt ab.

- In den meisten Fällen kann man es sich nicht erlauben, keine erfolgreiche Lehre vorweisen zu können. Aber werden Berufungskommissionen übermäBig enthusiastisch reagieren, wenn Antragssteller ihre Karriere für Lehrverbesserungen und -innovationen verwendet haben auf Kosten, zum Beispiel, ihrer Forschung oder der erbrachten Dienstleistungen? Abermals: wahrscheinlich nicht.

- In Australien sind Belege über Erfolge in der Akquisition und in der Durchführung von geförderten Forschungsvorhaben unerlässlich. Wer nicht als Hauptverantwortlicher in Drittmittelprojekten in Erscheinung getreten ist, wird kaum die Berufungsanforderungen erfüllen. Aber: der Grenznutzen von zusätzlich eingeworbenen Drittmitteln nimmt ab.

- Dienstleistungen in der Universität und der wissenschaftlichen Gemeinschaft sind $\mathrm{zu}$ erbringen. Akademiker müssen ihre Dienste für Zeitschriftengremien, Konferenzen und Universitätsgremien anbieten. Was kann erreicht werden, wenn man in vielen die- ser Gremien sitzt? Wenn Programmkomitees von über 50 Workshops genannt werden? Sehr wenig. Strategisch gesehen gilt: weniger ist mehr und die Qualität der Rollen (und der Gremien) sollte die Quantität dominieren.

Was sind nun dann die Motivationsfaktoren? Meiner Meinung nach sollten zumindest die Folgenden genannt werden:

- Zusammenarbeit mit der Wirtschaft: Wie bereits in den Beiträgen in Loos et al. (2013) festgestellt, nimmt die Bedeutung erfolgreicher Zusammenarbeit, Forschungskooperationen oder anderer Formen der Interaktion mit der Wirtschaft im Portfolio von Professoren $\mathrm{zu}$.

- Betreuung von Promotionsvorhaben: In Australien ist die Betreuung von Doktoranden nicht notwendigerweise abhängig von der Seniorität der Position, sondern vornehmlich von der Absolvierung von Betreuungstraining. Dies erlaubt es auch jungen Akademikern, an der (Ko-)Betreuung von Doktoranden teilzunehmen. Und die Anzahl erfolgreicher Betreuungen gilt als anerkanntes Kriterium für die Fähigkeit eines Akademikers, Forschung zu vermitteln und die Kapazitäten anderer zu fördern.

- Originalität des Forschungsvorhabens: Meiner Meinung nach ist das geplante Forschungsprogramm ein unterbewertetes Kriterium. Was wird der Forschungsfokus eines Akademikers in den nächsten 5 bis 10 Jahren sein? Wie hoch ist die Wahrscheinlichkeit, signifikante Ergebnisse und weitreichende Entwicklungen in der For- schung voranzutreiben? Die NeuroISEntwicklung (Dimoka et al. 2012) ist ein gelungenes Beispiel, wie junge Akademiker eine neue Forschungsrichtung in der Wirtschaftsinformatik definieren und aufbauen.

Natürlich gibt es noch weitere Faktoren, die ich hier nicht berücksichtigt habe. Dennoch bleibt die Unterscheidung in die Faktorkategorien hilfreich, um eine Strategie für die Karriereplanung zu entwickeln. Vereinfacht ausgedrückt könnte eine derartige Strategie wie folgt aussehen. Erstens: Hygienefaktoren sichern. Wenn manche Fähigkeiten noch nicht existieren (bspw. publizieren in TopJournals), sollten diese besser früher als später erlernt werden. Denn egal, was man glaubt, man wird nie wieder so viel Zeit zum Lernen neuer Fähigkeiten haben wie jetzt. Zweitens: Interdependenzen zwischen Hygienefaktoren verstehen. Beispielsweise können Lehre und Forschung koexistieren, aber erfolgreiche Drittmitteleinwerbung ist meiner Erfahrung nach abhängig von Publikationserfolgen und nicht umgekehrt. Zu guter Letzt: Hygienefaktoren vergessen. Wenn die relevanten Kriterien erfüllt (nicht übererfüllt!) sind, muss sich die Strategie auf die Motivationsfaktoren richten. Wie sieht das Forschungsprogramm aus? Wofür wird man in fünf Jahren bekannt sein? Kann man seine eigenen Erfolge (Publikationen) auf Andere (Doktoranden) ausweiten? Wie können Industriekooperationen aufgebaut werden?

Ich hoffe, dass die dargelegten Ideen und Sichtweisen zu dem konstruktiven Dialog über wissenschaftliche Karrierepfade beitragen und Interessierte da- 
durch in Ihrer persönlichen Strategieentwicklung unterstützt werden.

\section{Literatur}

Dean DL, Lowry PB, Humpherys SL (2011) Profiling the research productivity of tenured information systems faculty at US institutions. MIS Ouarterly 35(1):1-15

Dimoka A, Banker RD, Benbasat I, Davis FD, Dennis AR, Gefen D, Gupta A, Ischebeck A,
Kenning PH, Pavlou PA, Muller-Putz G, Ried $R$, vom Brocke J, Weber B (2012) On the use of neuropyhsiological tools in IS research: developing a research agenda for NeurolS. MIS Quarterly 36(3):679-702

Harzing A-W (2013) A preliminary test of Google scholar as a source for citation data: a longitudinal study of Nobel prize winners. Scientometrics 93(3):1057-1075

Herzberg $F$ (1966) Work and the nature of man. World Publishing, Cleveland

Lamp J (2009) Journal ranking and the dreams of academics. Online Information Review 33(4):827-830
Loos $\mathrm{P}$, Mertens $\mathrm{P}$, Eymann T, Hirschheim R, Schwenker B, Hess T (2013) Qualifikationsprofil für Universitätsprofessoren der Wirtschaftsinformatik. WIRTSCHAFTSINFORMATIK 5(2):107-114

Recker J, Young R, Darroch F, Marshall P, McKay J (2009) ACIS 2007 panel report: lack of relevance in IS research. Comm AIS 24(18):303-314

Rosemann M, Recker J, Vessey I (2010) An examination of IS conference reviewing practices. Comm AIS 26(15):287-304 